\title{
Suppression of Dollar Spot by Hypovirulent Isolates of Sclerotinia homoeocarpa
}

\author{
Ting Zhou and Greg J. Boland
}

First author: Agriculture \& Agri-Food Canada, Southern Crop Protection and Food Research Centre, 4902 Victoria Avenue N., Vineland Station, Ontario, LOR 2E0, Canada; and second author: Department of Environmental Biology, University of Guelph, Guelph, Ontario, N1G 2W1, Canada.

Accepted for publication 30 April 1998.

\begin{abstract}
Zhou, T., and Boland, G. J. 1998. Suppression of dollar spot by hypovirulent isolates of Sclerotinia homoeocarpa. Phytopathology 88:788-794.

Selected hypovirulent isolates of Sclerotinia homoeocarpa were evaluated for efficacy in suppressing dollar spot of turfgrass under growth room and field conditions. Under growth room conditions, hypovirulent isolates Sh12B, Sh09B, or Sh08D of S. homoeocarpa caused 3.4 to $30.4 \%$ diseased turf in comparison to virulent isolates Sh48B and Sh14D, which caused 80.2 to $90.2 \%$ disease. In treatments that received both virulent and hypovirulent isolates, only hypovirulent isolate Sh12B significantly reduced disease as compared with the control with virulent isolates alone. In a field experiment in 1993 on swards of creeping bentgrass artificially inoculated with a virulent isolate of the pathogen, all treatments containing hypovirulent isolate Sh12B applied as a mycelial suspension, granular mix, or alginate pellets developed significantly less disease (6.3 to $20.8 \%$

disease suppression was still significant 1 year after application when compared with the water control. Applications of hypovirulent isolate Sh09B did not reduce dollar spot in any treatments. Significant suppression of dollar spot by isolate Sh12B was also observed in the experiment conducted in 1994. In addition, suppression of dollar spot by hypovirulent isolate Sh12B was evaluated on swards with naturally occurring inoculum during 1994. Treatments with a mycelial suspension and alginate pellets of hypovirulent isolate Sh12B significantly reduced dollar spot compared with their respective formulation controls. With few exceptions, there was no statistical difference between treatments with hypovirulent isolate Sh12B and the fungicide chlorothalonil (Daconil 2787). Multiple applications of the hypovirulent isolate did not result in greater suppression of dollar spot as compared with a single application. The results indicate that hypovirulence has potential as an effective strategy for the management of dollar spot.
\end{abstract} diseased turf) compared with their respective formulation controls (23.8 to $31.2 \%$ ). Suppression of dollar spot by treatment with mycelial suspensions of isolate Sh12B was evident up to 45 days postinoculation, and
Additional keywords: dsRNA, turfgrass disease.
Dollar spot is a common and persistent disease of most turfgrasses. In North America, creeping bentgrass (Agrostis palustris Hudson) and annual bluegrass (Poa annua L.) are the turf species most susceptible to the disease. Patches of dollar spot on closely mown grasses are generally small and rarely exceed $6 \mathrm{~cm}$ in diameter. However, disease can be more serious and persistent on golf course putting greens, closely mown fairways, bowling greens, and home lawns (36). The causal agent of this disease is Sclerotinia homoeocarpa F.T. Bennett (4), but it may be more accurately classified within the genera Lanzia Sacc. or Moellerodiscus Henn. (20). The fungus does not form sclerotia, but produces plate-like black stromata and survives unfavorable periods as mycelia in infected plant tissues and as stroma on foliage (32). Although infertile aborted apothecial primordia have been produced by the fungus in culture and fertile apothecia have been observed from Festuca turf in field conditions (3), neither sexual nor asexual spores are considered important in the epidemiology of the disease $(10,19)$.

Management of dollar spot relies on increasing nitrogen fertilization, avoiding drought stress, avoiding or removing dew and guttation fluid, and, in many cases, applying fungicides (32). Although disease can be controlled by applications of fungicides throughout the year, these applications are being discouraged because of public concerns regarding the use of pesticides. The need for reduced pesticide use on golf courses is considered particularly important because of the often prolonged exposure of players to treated turf.

Corresponding author: T. Zhou; E-mail address: zhout@em.agr.ca

Publication no. P-1998-0618-01R

(c) 1998 Department of Agriculture and Agri-Food, Government of Canada
In addition, the annual cost of fungicide applications often exceeds $\$ 170$ per ha in southern Ontario and the United States. More money is spent to manage dollar spot than any other disease in golf courses (13). Moreover, in some areas, strains of S. homoeocarpa have developed resistance to selected fungicides (12). Thus, more environmentally acceptable strategies for the management of dollar spot are needed.

Alternative methods to the use of fungicides are being investigated for the management of dollar spot, and biological control has shown promise. Fungal antagonists such as Fusarium heterosporum Nees:Fr. and Gliocladium virens J.H. Miller, J.E. Giddens, \& A.A. Foster have been shown to suppress dollar spot by up to 93 and $70 \%$, respectively, when applied as inundative treatments in specific formulations $(13,14)$. Recently, the biological fungicide, BIO-TREK (BioWorks, Inc., Geneva, NY), containing Trichoderma harzianum Rifai, was registered with the U.S. Environmental Protection Agency for control of fungal diseases of turf including dollar spot $(23,24)$. Applications of bacteria such as Enterobacter cloacae to bentgrass putting greens naturally infested with $S$. homoeocarpa provided up to $63 \%$ disease control, and suppression was evident for up to 2 months following application (27). Additional bacteria such as Pseudomonas spp. and Streptomyces hygroscopicus have been evaluated for efficacy in the phyllosphere of turf $(17,31)$. Applications of compost-amended topdressings and other amendments have also shown promise for suppression of dollar spot, and this efficacy may, at least in part, be attributable to microbial suppression of disease $(22,28)$.

Suppression of fungal plant diseases by hypovirulent isolates of plant pathogens was first reported for chestnut blight, caused by Cryphonectria parasitica (Murrill) Barr (35). In Europe and some 
regions of the United States, the introduction of hypovirulent isolates has successfully controlled chestnut blight (25). Hypovirulence also has been reported in other plant pathogens. A hypovirulent isolate of Rhizoctonia solani Kühn AG-4 (isolate 521) significantly reduced damping-off of cotton and radish seedlings (18). In controlled environments, mycelial suspensions of a hypovirulent isolate of $S$. minor Jagger applied to leaf lesions initiated by compatible virulent isolates on lettuce suppressed lesion expansion by up to $100 \%$ (26). Application of a hypovirulent strain of Gaeumannomyces graminis var. tritici in field trials reduced crown root infection of winter wheat and increased yield of spring wheat by $20.8 \%(8)$.

Several isolates of $S$. homoeocarpa that are hypovirulent on creeping bentgrass have been identified (37). Hypovirulence in isolate Sh12B was associated with the presence of double-stranded ribonucleic acid (dsRNA), which is indicative of the presence of a fungal virus, virus-like agent, or hypovirus $(16,29,30)$. More importantly, the hypovirulent phenotype and dsRNA were transmitted from isolate Sh12B to several recipient, virulent isolates through hyphal anastomosis. Coinoculation of detached leaves of creeping bentgrass with virulent isolate $\mathrm{Sh} 48 \mathrm{~B}$ and hypovirulent isolate Sh12B resulted in significant reductions in lesion length compared with inoculation with Sh48B alone (39). This preliminary identification of hypovirulent isolates of $S$. homoeocarpa presented an opportunity in the current study to evaluate the potential use of hypovirulence for disease management of dollar spot. The objectives of this study were to evaluate the efficacy of selected hypovirulent isolates for suppression of dollar spot under controlled environment and field conditions and to determine the influence of application methods and frequency on the effectiveness of disease suppression.

\section{MATERIALS AND METHODS}

Fungal isolates. Isolates Sh48B and Sh14D of S. homoeocarpa, obtained from Festuca spp. and A. palustris, respectively, were selected as typical virulent isolates according to culture morphology and pathogenicity on creeping bentgrass. Isolates Sh09B, Sh12B, and Sh08D from Festuca spp. were hypovirulent isolates containing dsRNA (39). All isolates were stored at $4^{\circ} \mathrm{C}$ on oatmeal sand medium (OSM: oatmeal, $30 \mathrm{ml}$; sand, $60 \mathrm{ml}$; and distilled water, $10 \mathrm{ml}$; autoclaved at $121^{\circ} \mathrm{C}$ for $40 \mathrm{~min}$ ) modified from Goodman and Burpee (13). Isolates were subcultured on potato dextrose agar (PDA: potato infusion, $200 \mathrm{~g} /$ liter; dextrose, $20 \mathrm{~g} / \mathrm{liter}$; and agar, $15 \mathrm{~g} /$ liter) at $22^{\circ} \mathrm{C}$ for 5 to 7 or 10 to 14 days for virulent or hypovirulent isolates, respectively, before further use.

Formulations. Mycelial suspensions, granular mixes, and alginate pellets were prepared. For mycelial suspensions, individual isolates were cultured in potato dextrose broth (PDB: potato infusion, $200 \mathrm{~g} /$ liter, and dextrose, $20 \mathrm{~g} / \mathrm{liter}$ ) on a shaker (Lab-line Orbit Shaker; Lab-line Instruments, Inc., Melrose Park, IL) at $150 \mathrm{rpm}$ for 10 to 14 days at room temperature $\left(20\right.$ to $\left.22^{\circ} \mathrm{C}\right)$. The resulting mycelial mass was harvested by vacuum filtration using a Whatman \#3 filter paper (Whatman International, Ltd., Maidstone, England), blended in a commercial blender for $30 \mathrm{~s}$, and resuspended in sterile distilled water to give a concentration of $10 \%$ mycelium (vol/vol). For the granular mixes, selected isolates were cultured on PDA for 5 to 10 days. Plugs of PDA ( $5 \mathrm{~mm}$ diameter) colonized by the fungal isolates were used to inoculate flasks $(250 \mathrm{ml})$ containing $100 \mathrm{ml}$ of OSM. Each flask was inoculated with 10 plugs of PDA and incubated at 20 to $22^{\circ} \mathrm{C}$ for 2 to 4 weeks. Colonized OSM was air-dried in a laminar flow transfer chamber and ground with a mortar and pestle. The resulting granular mix was sieved through a 1-mm screen prior to use. For alginate pellets, sodium alginate (BDH Inc., Toronto, Ontario, Canada) and ground oatmeal were added to a mycelial suspension (10\% mycelium, $\mathrm{vol} / \mathrm{vol}$ ) prepared as described above and mixed in a blender. The final mix contained $1 \%$ sodium alginate $(\mathrm{wt} / \mathrm{vol})$ and $10 \%$ oatmeal (wt/vol). Pellets were prepared by dropping the mix into a $0.1 \mathrm{M}$ calcium chloride solution (Fisher Scientific Co., Fair Lawn, NJ) and then air-dried for $24 \mathrm{~h}$ at 20 to $22^{\circ} \mathrm{C}$ in a laminar flow transfer chamber. To verify the viability of $S$. homoeocarpa in pellets, 30 pellets were transferred onto each of two water agar (1\%) plates, and the number of pellets that formed colonies of $S$. homoeocarpa was determined.

Host plants. Creeping bentgrass was used in both growth room and field experiments. In the growth room $\left(22 / 18^{\circ} \mathrm{C}\right.$ day/night; $16-\mathrm{h}$ photoperiod), grass seed (cv. Cobra, approximately four seeds per $\mathrm{cm}^{2}$ ) was sown in pasteurized greenhouse soil mix (Sunshine Mix 4; Sun Gro Horticulture Canada Ltd., Bellevue, WA) in plastic pots $(7.5 \times 7.5 \times 10 \mathrm{~cm})$ placed in a plastic tray. The grass in the pots was watered every other day, fertilized with $0.2 \%$ N-P-K (2020-20) biweekly, and cut to a height of $2 \mathrm{~cm}$ weekly using handheld electric sheers. Pots of 4- to 6-week-old grass (approximately $50 \mathrm{~cm}^{2}$ of grass per pot) were used in all experiments.

All field experiments were conducted at the Cambridge Research Station, Cambridge, Ontario, Canada, on an established sward of creeping bentgrass ('Cobra') maintained as recommended for golf course putting greens (2), except for applications of fertilizer that were reduced by approximately $50 \%$ to encourage disease development.

Growth room experiments. An experiment was conducted to evaluate the efficacy of selected hypovirulent isolates in the suppression of dollar spot. Individual pots of grass were treated with inoculum of virulent isolates, hypovirulent isolates, or both. Two virulent isolates, Sh48B and Sh14D, were applied individually to pots as a granular mix at a rate of $0.4 \mathrm{~g}$ per pot. Three hypovirulent isolates, Sh09B, Sh12B, and Sh08D, were applied individually to pots as alginate pellets at a rate of $0.1 \mathrm{~g}$ per pot. Formulation control treatments included alginate pellets containing no fungal mycelium. Treated pots were arranged in plastic trays in a completely randomized design with four replications (pots) per treatment, and the experiment was repeated once. The trays were covered with a transparent plastic cover to maintain moisture for $48 \mathrm{~h}$ after inoculation. The percentage of turf area in each pot with symptoms of dollar spot (percent diseased turf) was evaluated visually 7 to 8 days after treatment (13).

A second experiment was conducted to determine the effect of inoculum formulations and application frequency on suppression of dollar spot. All pots of grass, except for the untreated controls, were top-dressed with virulent isolate Sh48B as a granular mix at a rate of $0.4 \mathrm{~g}$ per pot. Hypovirulent isolates were applied by spraying mycelial suspensions at a rate of $1 \mathrm{ml}$ per pot, or by top-dressing alginate pellets or granular mix at a rate of $0.1 \mathrm{~g}$ per pot. Individual pots of grass were treated with hypovirulent isolates once (immediately after inoculation with virulent isolate Sh48B), twice (immediately after inoculation and 1 week later), or three times (immediately after inoculation, and 1 week and 2 weeks later). Grass treated with water, alginate pellets, or granular mix without the hypovirulent isolate were used as formulation controls. The experiment was arranged in a completely randomized design with four replications (pots) per treatment and was repeated once. All treated pots of grass were maintained as described above. The percentage of turf area per pot with symptoms of dollar spot (percent diseased turf) was evaluated visually 4 weeks after inoculation with the virulent isolate.

Field experiments with artificial inoculation. Experiments were conducted in 1993 and 1994 on swards of creeping bentgrass with a history of dollar spot. Both experiments were arranged in a randomized complete block design with four replications per treatment. To increase the amount of inoculum, all treatments except the untreated control were inoculated by top-dressing with virulent isolate $\mathrm{Sh} 48 \mathrm{~B}$ as a granular mix at a rate of $10 \mathrm{~g} / \mathrm{m}^{2}$. In 1993 , individual plots were $0.25 \mathrm{~m}^{2}$, with a $0.5-\mathrm{m}$ border between plots. Two hypovirulent isolates, Sh09B and Sh12B, were applied in three formulations immediately after inoculation with virulent isolate 
Sh48B. Mycelial suspensions were applied at a rate of $80 \mathrm{ml} / \mathrm{m}^{2}$ and were sprayed with a hand-sprayer (Mr. Mister; BDR Products, Inc., Oakville, Ontario, Canada) after dilution with distilled water to $200 \mathrm{ml} / \mathrm{m}^{2}$ for improved distribution. The granular mix and alginate pellets were applied by hand as topdressings at a rate of $8 \mathrm{~g} / \mathrm{m}^{2}$. All treatments were applied on 20 August 1993. The percentage of turf area per plot with symptoms of dollar spot (percent diseased turf) was assessed visually (13) at 6- to 10-day intervals in 1993 and again during July to September 1994.

In 1994, individual plots were $1 \mathrm{~m}^{2}$, with a 1 -m border between plots. Only hypovirulent isolate Sh12B was evaluated, because of the poor performance of Sh09B in the growth room experiments and in the 1993 field experiment. Mycelial suspensions were applied at a rate of $100 \mathrm{ml} / \mathrm{m}^{2}$ and were diluted with distilled water to $200 \mathrm{ml} / \mathrm{m}^{2}$ before application with a hand-sprayer. The granular mix and alginate pellets were applied by hand as topdressings at a rate of $10 \mathrm{~g} / \mathrm{m}^{2}$. All treatments were applied on 26 May 1994. Disease assessment was conducted as in the 1993 experiment.

Field experiment with naturally occurring inoculum. To determine the effects of a hypovirulent isolate on suppression of naturally occurring dollar spot, an experiment was established on a sward of creeping bentgrass with a history of severe dollar spot. The experiment was initiated in June 1994 when dollar spot first appeared and ended in October 1994 when disease development stopped. Individual plots were $2 \mathrm{~m}^{2}$, with a 1 -m border between plots. Treatments included hypovirulent isolate Sh12B applied as a mycelial suspension $\left(100 \mathrm{ml} / \mathrm{m}^{2}\right)$ or as alginate pellets $\left(10 \mathrm{~g} / \mathrm{m}^{2}\right)$, and the fungicide $\left(1 \mathrm{ml} / \mathrm{m}^{2}\right)$ chlorothalonil (40.4\%) (Daconil 2787; ISK BioSciences, Mentor, $\mathrm{OH}$ ). Treatments were applied once (10 June), twice (10 June and 12 August), or four times (10 June, 8 July, 2 August, and 11 September). Control treatments included water and alginate pellets without Sh12B and were applied four times. The mycelial suspension and fungicide were diluted with distilled water to $200 \mathrm{ml} / \mathrm{m}^{2}$ before application with a $\mathrm{CO}_{2}$-sprayer (R\&D Sprayers, Inc., Opelousas, LA). Treatments were arranged as a randomized complete block design with four replications per treatment. Disease assessment was conducted as in the 1993 field experiment.

Data analysis. Data were analyzed using the General Linear Models procedure of SAS/STAT 6.11 (SAS Institute, Inc., Cary, NC). Percentage data, except for the field experiment with naturally occurring inoculum, were transformed to arcsine values before analyses of variance (21). Differences among or between treatments were determined by orthogonal contrasts or Fisher's protected least

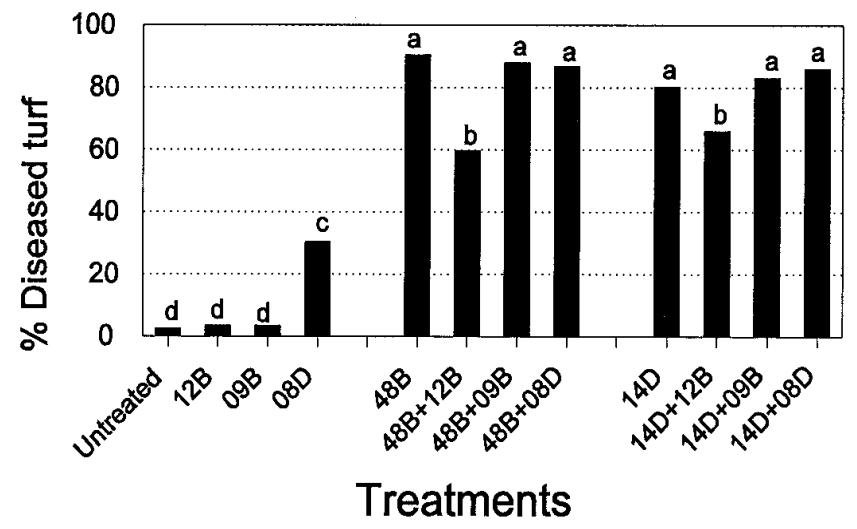

Fig. 1. Percentage of diseased creeping bentgrass turf 8 days after treatment with selected virulent and hypovirulent isolates of Sclerotinia homoeocarpa in growth room experiments. Treatments included isolates applied individually or as a coinoculum mixture. Hypovirulent isolates Sh12B, Sh09B, and Sh08D were applied as alginate pellets, and virulent isolates Sh48B and Sh14D were applied as a granular mix. Untreated = uninoculated control. Treatments followed by the same letter are not significantly different according to Fisher's protected least significant difference test $(P=0.05)$. significant difference test. Data from experimental repetitions were tested for homogeneity of variances prior to pooling (21).

\section{RESULTS}

Growth room evaluation of hypovirulent isolates. In the growth room experiments, hypovirulent isolates Sh12B, Sh09B, or Sh08D caused significantly less disease (3.4 to $30.4 \%$ diseased turf) than virulent isolates Sh48B or Sh14D (80.2 to $90.5 \%$ diseased turf) (Fig. 1). The percent diseased turf caused by hypovirulent isolates Sh09B and Sh12B was not significantly different from the untreated control. Hypovirulent isolate Sh08D caused less disease than virulent isolates Sh48B and Sh14D, but more than hypovirulent isolates Sh12B and Sh09B. Isolate Sh12B was the only hypovirulent isolate that significantly reduced disease ratings compared with the controls treated only with the virulent isolates. Similar results were obtained when the experiment was repeated, but the variances of the two experiments were not homogenous and, therefore, could not be combined (21). Thus, only one representative experiment is shown in Figure 1.

Growth room evaluation of formulations of hypovirulent isolates. The results of this experiment were presented as simple effects in Figure 2, because the interactions of formulations and application times were significantly different $(P<0.01)(21)$. The three formulations of isolate Sh12B differed in their ability to suppress dollar spot $(P<0.01)$. All treatments with mycelial suspensions of Sh12B (applied once, twice, or three times) had significantly lower percent diseased turf (7.5 to $15.0 \%)$ as compared with the water control $(42.5 \%)$. There were no significant differences among the three mycelial suspension treatments. As compared with the granular mix control $(57.5 \%)$, the percent diseased turf was significantly lower in the treatment with granular mix of Sh12B applied two and three times (18.8 and $35.5 \%$, respectively), but disease was not significantly lower when the granular mix was applied once. The percent diseased turf in all treatments with pellets of Sh12B (30.0 to 42\%) was similar to the pellet control (45.0\%). In a repeated experiment, as compared with their formulation controls, significantly lower disease ratings were obtained in all three

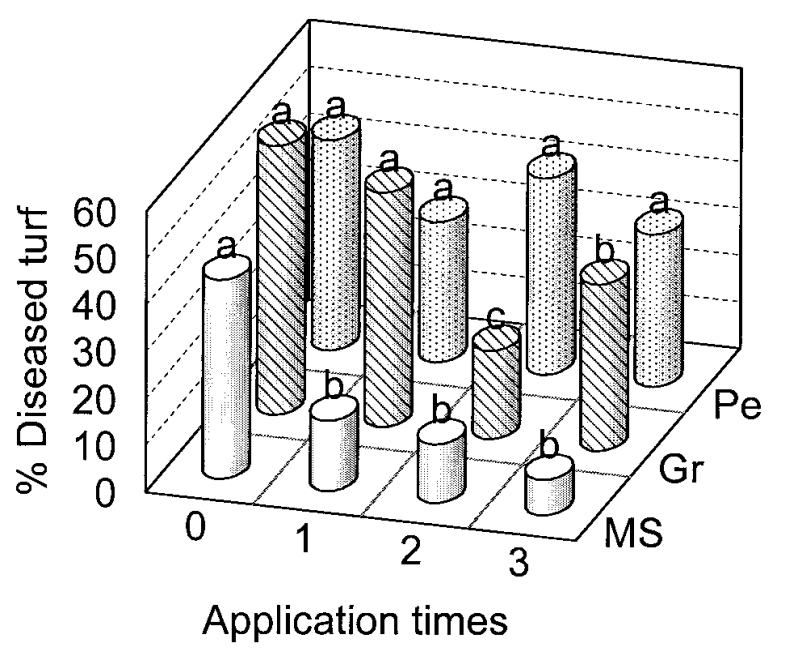

Fig. 2. Percentage of diseased creeping bentgrass turf 4 weeks after inoculation with virulent and hypovirulent isolates of Sclerotinia homoeocarpa in growth room experiments. Treatments included hypovirulent isolate Sh12B applied in different formulations: $\mathrm{MS}=$ mycelial suspension; $\mathrm{Gr}=$ ground oatmeal sand medium colonized with $\mathrm{Sh} 12 \mathrm{~B}$; and $\mathrm{Pe}=$ alginate pellets containing hyphal fragments of Sh12B. $0=$ formulation control without isolate Sh12B; and 1, 2, and 3 = grass treated with isolate Sh12B one, two, and three times at 7-day intervals. All pots of grass were top-dressed with virulent isolate $\mathrm{Sh} 48 \mathrm{~B}$ in a granular mix. Treatments within rows (application times) followed by the same letter are not significantly different according to orthogonal contrast comparisons $(P=0.05)$. 
mycelial suspension treatments, but significantly different disease ratings were not detected with either granular mix or alginate pellets (data not shown).

Field experiments with artificial inoculation. In the field experiment in 1993, at 10 days after treatment (30 August), the percent diseased turf for each formulation of hypovirulent isolate Sh12B was $6.3,12.5$, and $20.8 \%$ for treatments applied as a mycelial suspension, granular mix, and alginate pellets, respectively, and were significantly lower than their respective formulation controls (31.2, 23.8 , and $30.0 \%$, respectively). Suppression of dollar spot by the mycelial suspension of isolate Sh12B was still evident 45 days after treatment (4 October), but the granular mix and alginate pellets were ineffective at this time (Fig. 3). Turf inoculated with virulent isolate Sh48B had significantly higher disease ratings than the uninoculated control at 10 and 45 days postinoculation. Disease ratings of the pellet control were similar to the water control, but disease ratings of the granular mix control were slightly lower than the water control. Applications of hypovirulent isolate Sh09B did not reduce dollar spot in any treatments.

When the experimental plots were assessed in summer 1994, the amount of dollar spot in the plot treated with isolate Sh12B in 1993 was still significantly less on all five disease rating dates compared with the water control treatment (Fig. 4). For example, on 19 August, exactly 1 year after application of the treatments, the percent diseased turf was $35.0,31.3$, and $40.0 \%$ in plots treated with isolate Sh12B as a mycelial suspension, alginate pellets, and granular mix, respectively, but was $61.3 \%$ in the water control.

Isolate Sh12B was evaluated using larger plots in 1994. The results of the 1994 experiment (Fig. 5) were similar to those obtained in 1993 (Fig. 3). When disease was assessed 12 days (7 June) after treatment, plots that were treated with isolate Sh12B applied as a mycelial suspension, granular mix, and alginate pellets had $15.8,18.8$, and $22.5 \%$ diseased turf, respectively, whereas their respective formulation controls had $31.3,40.0$, and $36.3 \%$ disease, respectively (Fig. 5). Plots treated with pellets containing isolate Sh12B had lower disease ratings than the pellet formulation control. When plots were assessed at 60 days ( 25 July) after treatment, the percent diseased turf increased to 77.5 to $85.0 \%$ in the three formulation controls, and all treatments containing isolate Sh12B had significantly less disease (47.5 to $60.0 \%$ ).

Field experiment with naturally occurring inoculum. In 1994, the ability of hypovirulent isolate Sh12B to suppress dollar spot on swards resulting from naturally occurring inoculum was evalu-

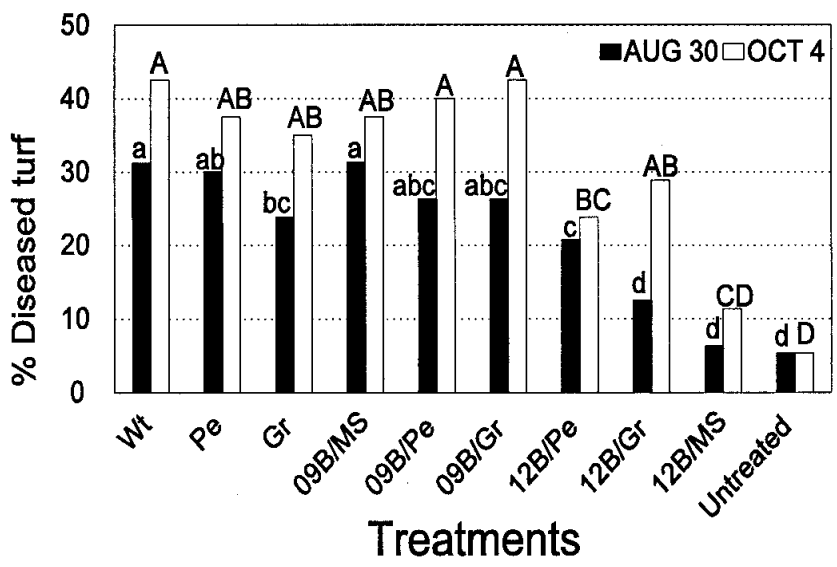

Fig. 3. Percentage of diseased creeping bentgrass turf in the 1993 field experiment. Plots were inoculated with virulent isolate Sh48B and treated with hypovirulent isolates of Sclerotinia homoeocarpa on 20 August 1993. Wt = water control; $\mathrm{MS}=$ mycelial suspension; $\mathrm{Pe}=$ alginate pellet $; \mathrm{Gr}=$ granular mix of oatmeal sand medium; 09B and 12B = hypovirulent isolates Sh09B and Sh12B, respectively; and Untreated $=$ uninoculated control. Treatments followed by the same lower (30 August 1993) or upper (4 October 1993) case letter are not significantly different according to Fisher's protected least significant difference test $(P=0.05)$. ated. Figure 6 summarizes the percent diseased turf 1 week after the second (15 July), third (19 August), and fourth (18 September) applications and at the end of the season (15 October). No data were collected after the first application in June, because little disease was observed. When the data were analyzed as a factorial design, the interactions of treatments and times of application were significantly different $(P=0.05)$ on at least one date of assessment (15 July). Therefore, results were presented as simple effects (21). All treatments with mycelial suspensions of isolate Sh12B had significantly less disease compared with the water control. With few exceptions, disease ratings of treatments with isolate Sh12B applied as pellets were lower than the water or pellet control (Fig. 6). All disease ratings in plots treated with the mycelial suspension of isolate Sh12B were similar to disease ratings in plots treated with the fungicide Daconil 2787, except for the treatments with four applications assessed on 15 July and 15 October (Fig. 6A and D). For treatments with four applications, plots treated with the fungicide had a lower disease ratings than plots with isolate Sh12B in alginate pellets. However, there were no significant

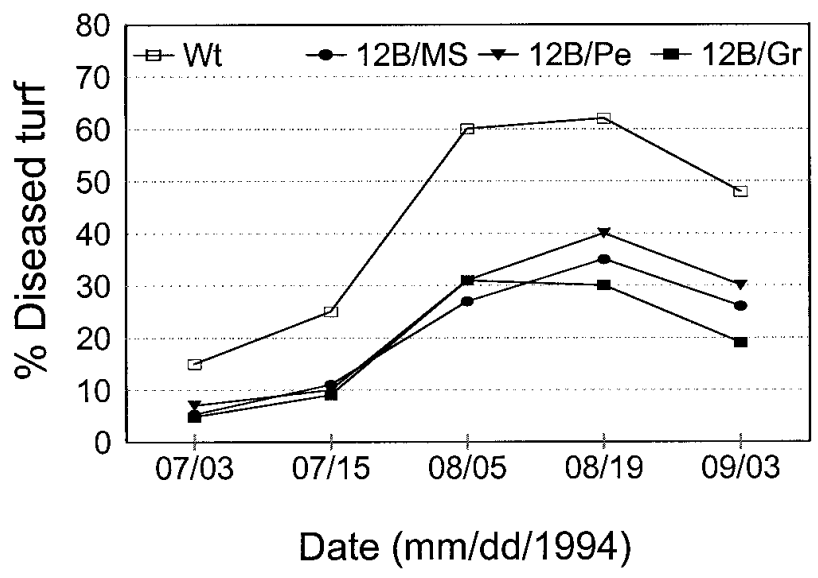

Fig. 4. Persistence of dollar spot suppression in 1994 by application of hypovirulent isolate Sh12B of Sclerotinia homoeocarpa in field plots inoculated with virulent isolate Sh48B on 20 August 1993. Percent diseased turf is the percentage of the plot area of creeping bentgrass with symptoms of dollar spot. $\mathrm{Wt}=$ water control, $\mathrm{MS}=$ mycelial suspension, $\mathrm{Pe}=$ alginate pellet, and $\mathrm{Gr}=$ granular mix. Data were analyzed for individual disease assessment dates. Treatments containing isolate Sh12B are significantly different from the water control, but are not significantly different from each other in all disease assessment dates, according to orthogonal contrast comparisons $(P=0.05)$.

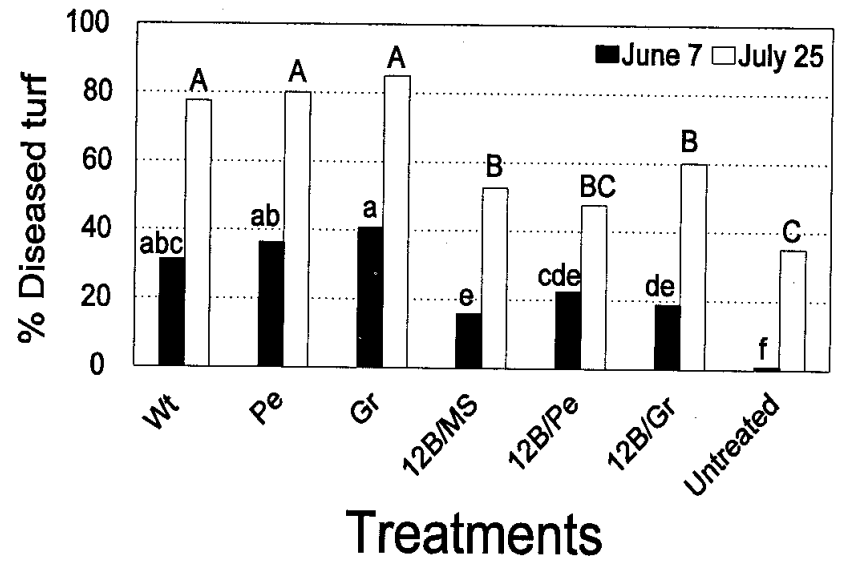

Fig. 5. Percentage of diseased creeping bentgrass turf after application with hypovirulent isolate Sh12B of Sclerotinia homoeocarpa in field plots inoculated with virulent isolate Sh48B on 26 May 1994. Wt $=$ water control, MS = mycelial suspension, $\mathrm{Pe}=$ alginate pellet, $\mathrm{Gr}=$ granular mix , and Untreated = uninoculated control. Treatments followed by the same lower (7 June 1994) or upper (25 July 1994) case letter are not significantly different according to Fisher's protected least significant difference test $(P=0.05)$. 
differences between treatments of fungicide and pellets of isolate Sh12B when they were applied once or twice. For all treatments with isolate Sh12B, there were no statistical differences either between the mycelial suspension and alginate pellets, or among the treatments applied once, twice, or three times.

\section{DISCUSSION}

Although hypovirulence has been reported in a number of fungal pathogens $(5,6,26,29,37)$, the application of hypovirulent isolates to suppress plant diseases has been effective in only a few pathosystems $(1,8,9,11,15)$. This study has demonstrated for the first time that hypovirulent isolates of $S$. homoeocarpa can be used to suppress dollar spot of creeping bentgrass under both controlled environment and field conditions. Hypovirulent isolate Sh12B applied as a mycelial suspension in the field suppressed dollar spot by up to $80 \%$. Disease suppression persisted until 1 year after application. These results provide evidence that hypovirulent isolates of $S$. homoeocarpa have potential as an effective disease management strategy for dollar spot of turfgrass.

The principle of using hypovirulent isolates of fungal plant pathogens to suppress plant disease is that the hypovirulent phenotype can be transmitted from an introduced hypovirulent isolate(s) to the wild-type pathogen population and, thereby, reduce the virulence of the population $(7,29,35)$. In addition to a reduction in virulence, hypovirulent isolates in many plant pathogens also often display reductions in growth, reproduction, and survival that may contribute to reductions in the fitness of the wild-type population. Our previous studies (39) established that the hypovirulent phenotype of isolate Sh12B can be transmitted in vitro and in vivo into several virulent isolates, including isolate Sh48B used in this study.
In a preliminary study using a semiselective medium to recover fungal isolates inoculated onto creeping bentgrass, transmission of the hypovirulent phenotype and dsRNA from isolate Sh12B into a virulent, dsRNA-negative, demethylation inhibitor-resistant isolate Ky-7 was evident in growth room experiments (M. Wang, T. Zhou, and G. Boland, unpublished data). However, attempts to transmit the hypovirulent phenotype and dsRNA from isolate Sh09B into virulent isolate Sh48B were unsuccessful (39), and applications of hypovirulent isolate Sh09B failed to reduce dollar spot in both controlled environment and field conditions.

Hypovirulent isolates, like other types of biocontrol agents, may induce plant resistance or produce unidentified compounds that inhibit the pathogen or interfere with virulent isolates for nutrients and infection sites $(11,15)$. Application of a hypovirulent isolate of $R$. solani reduced cotton seedling disease incited by $R$. solani. It was found that colonization of roots and hypocotyls by the hypovirulent isolate provided a physical barrier for the recognition of infection sites, which protected the host from infection by the pathogen (33). More intensive studies are needed to understand the mode of action involved in the suppression of dollar spot by hypovirulent isolate Sh12B.

Conversion of a pathogen population from virulent to hypovirulent through hyphal anastomosis appears to be one of the most important steps in biological control using hypovirulence (11). Although vegetative incompatibility is not a complete barrier to the transmission of hypovirulence (35), it is a key factor in permitting hyphal anastomoses between fungal isolates. In controlled environments, mycelial suspensions of a hypovirulent isolate of $S$. minor applied to leaf lesions initiated by a compatible virulent isolate on lettuce suppressed lesion expansion by up to $100 \%$, but no such reduction was obtained when an incompatible virulent

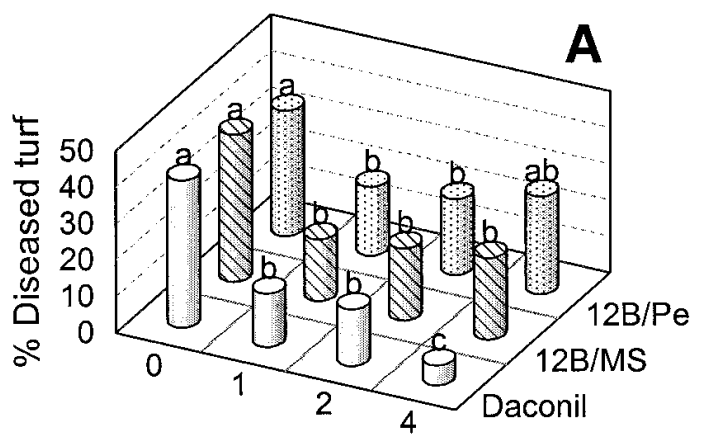

Application times

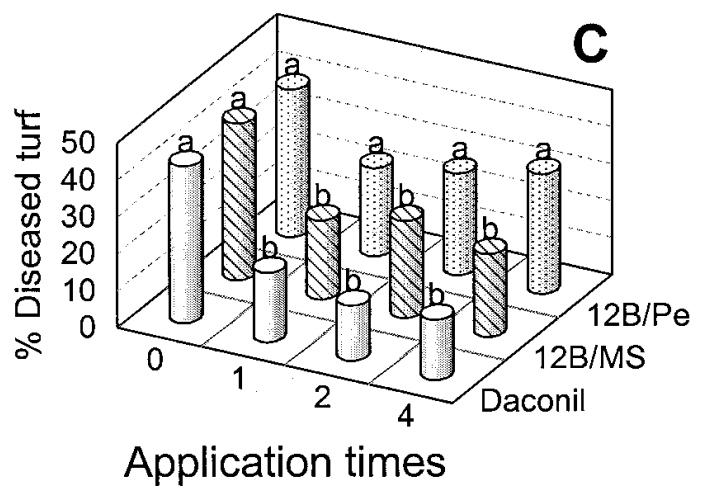

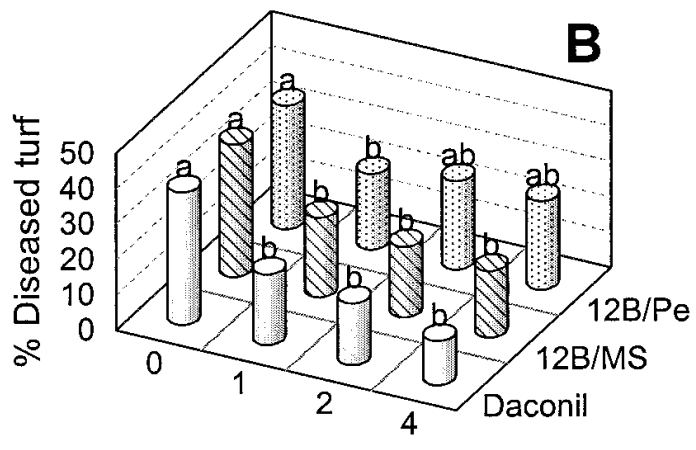

Application times

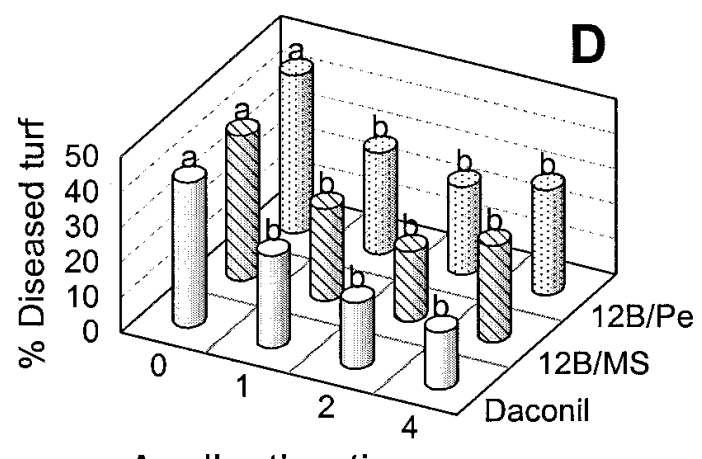

Application times



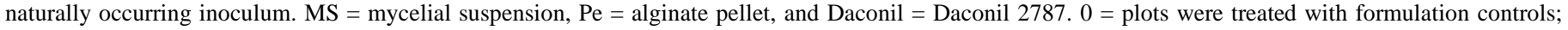

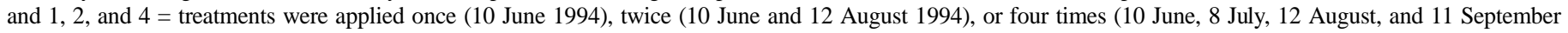

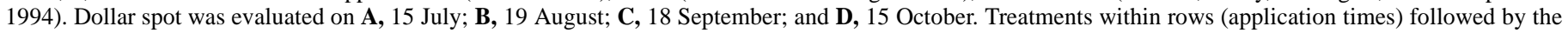
same letter are not significantly different according to orthogonal contrast comparisons $(P=0.05)$. 
isolate was used (26). Mycelial compatibility groups have been reported in populations of $S$. homoeocarpa $(34,38)$, but little information is available on the extent of such incompatibility in this fungus. Not all hypovirulent isolates used in our experiments were effective in suppression of dollar spot. It is possible that the failure of isolate Sh09B in disease suppression was due to vegetative incompatibility. Vegetative compatibility between isolate Sh09B and the virulent isolates involved in these experiments has not been determined, but should be clarified. Also, disease suppression in the experiment with naturally occurring inoculum of the pathogen was lower compared with that obtained with artificial inoculations. This may be due to the presence of more than one vegetative compatibility group in the populations of the pathogen in the experimental plots (38).

In general, the selected formulations of hypovirulent isolate Sh12B initially resulted in different degrees of disease suppression shortly after treatment, but the suppression levels were similar when disease was rated after a longer period. Mycelial suspensions seemed to be more effective than other formulations, but formulations were not directly compared because of differences in the amount of mycelia in the OSM compared with the alginate and mycelial formulations. The apparent improved efficacy of mycelial suspensions may have resulted from better and faster spatial coverage or improved contact with the virulent isolate. Although the same amount of mycelia was used in the mycelial suspensions and alginate pellets for each treatment, mycelia of the hypovirulent isolate that were encased in the dry pellets required additional time to absorb water and then grow out of the pellet to establish contact with the virulent isolates. Additives in the pellets, such as oatmeal, can influence the virulent and hypovirulent isolates, other microorganisms in the phyllosphere, and the final severity of dollar spot disease.

Multiple applications of hypovirulent isolate Sh12B did not result in higher disease suppression, which was evident in both growth room and field experiments. Hypovirulent isolates are not avirulent and can still cause disease although the disease is not severe, such as in the case of hypovirulent isolates of $C$. parasitica. Thus, the amount of inoculum applied and the time of application are important factors in utilizing hypovirulent isolates for disease management.

An improved understanding of the ecology of hypovirulent isolates of S. homoeocarpa and of the molecular ecology of the dsRNA agents associated with the hypovirulent phenotype would contribute to the potential development of this biological control strategy. However, hypovirulent isolates typically also display reductions in growth, reproduction, or survival that make their recovery more difficult than virulent isolates. In addition, methods to assess individual isolates for the presence of dsRNA are timeconsuming, labor-intensive, and expensive. Improved methods for the detection or recovery of virulent and hypovirulent isolates and the detection of specific segments of dsRNA in hypovirulent isolates or in planta would enable a more critical approach to the ecology and epidemiology of hypovirulence in dollar spot.

\section{ACKNOWLEDGMENTS}

We thank the Natural Sciences and Engineering Research Council of Canada (NSERC), the Ontario Ministry of Agriculture, Food and Rural Affairs (OMAFRA), and the Ontario Turfgrass Research Foundation (OTRF) for financial assistance. We also thank M. Wang and E. A. Smith for technical assistance.

\section{LITERATURE CITED}

1. Anagnostakis, S. L. 1982. Biological control of chestnut blight. Science 215:466-471.

2. Anonymous. 1993. Recommendations for Turfgrass Management. Publication 384. Ontario Ministry of Agriculture and Food, Toronto.

3. Baldwin, N. A., and Newell, A. J. 1992. Field production of fertile apothecia by Sclerotinia homoeocarpa in Festuca turf. J. Sports Turf Res. Inst. 68:73-76.
4. Bennett, F. T. 1937. Dollarspot disease of turf and its causal organism, Sclerotinia homoeocarpa N. sp. Ann. Appl. Biol. 24:236-257.

5. Boland, G. J. 1992. Hypovirulence and double stranded RNA in Sclerotinia sclerotiorum. Can. J. Plant Pathol. 14:10-17.

6. Buck, K. W. 1987. Viruses of plant pathogenic fungi. Pages 111-126 in: Genetics and Plant Pathogenesis. P. R. Day and G. J. Jellis, eds. Blackwell Scientific Publications, Oxford.

7. Dubos, B. 1987. Fungal antagonism in aerial agrobiocenoses. Pages 107135 in: Innovative Approaches to Plant Disease Control. I. Chet, ed. John Wiley \& Sons, New York.

8. Duffy, B. K., and Weller, D. M. 1996. Biological control of take-all of wheat in the pacific northwest of the USA using hypovirulent Gaeumannomyces graminis var. tritici and fluorescent Pseudomonads. J. Phytopathol. 144:585-590.

9. Elliston, J. E. 1982. Hypovirulence. Pages 1-33 in: Advances in Plant Pathology. Vol. 1. D. S. Ingram and P. H. Williams, eds. Academic Press, London.

10. Fenstermacher, J. M. 1979. Certain features of dollar spot disease and its causal organism, Sclerotinia homoeocarpa. Pages 49-53 in: Advances in Turfgrass Pathology. P. O. Larsen and B. G. Joyner, eds. HBJ, Duluth, MN.

11. Fulbright, D. W., Paul, C. P., and Garrod, S. W. 1988. Hypovirulence: A natural control of chestnut blight. Pages 121-139 in: Biocontrol of Plant Diseases. K. G. Mukerji and K. L. Garg, eds. CRC Press, Boca Raton, FL.

12. Golembiewski, R. C., Vargas, J. M., Jr., Jones, A. L., and Detweiler, A. R. 1995. Detection of demethylation inhibitor (DMI) resistance in Sclerotinia homoeocarpa populations. Plant Dis. 79:491-493.

13. Goodman, D. M., and Burpee, L. L. 1991. Biological control of dollar spot disease of creeping bentgrass. Phytopathology 81:1438-1446.

14. Haygood, R. A., and Mazur, A. R. 1990. Evaluation of Gliocladium virens as a biocontrol agent of dollar spot on Bermudagrass. (Abstr.) Phytopathology 80:435.

15. Herr, L. J. 1995. Biological control of Rhizoctonia solani by binucleate Rhizoctonia spp. and hypovirulent $R$. solani agents. Crop Prot. 14:179-186.

16. Hillman, B. I., Fulbright, D. W., Nuss, D. L., and van Alfen, N. K. 1995. Hypoviridae. Pages 261-264 in: Virus Taxonomy: Sixth Report of the International Committee for the Taxonomy of Viruses. F. A. Murphy, C. M. Fauquet, D. H. L. Bishop, S. A. Ghabrial, A. W. Jarvis, G. P. Martell, M. P. Mayo, and M. D. Summers, eds. Springer-Verlag, New York.

17. Hodges, C. F., Campbell, D. A., and Christians, N. 1993. Evaluation of Streptomyces for biocontrol of Bipolaris sorokiniana and Sclerotinia homoeocarpa on the phylloplane of Poa pratensis. J. Phytopathol. 139: 103-109.

18. Ichielevich-Auster, M., Sneh, B., Koltin, Y., and Barash, I. 1985. Suppression of damping-off caused by Rhizoctonia species by a nonpathogenic isolate of $R$. solani. Phytopathology 75:1080-1084.

19. Jackson, N. 1973. Apothecial production of Sclerotinia homoeocarpa F.T. Bennett. Pages 353-357 in: Proc. Int. Turfgrass Res. Conf. American Society of Agronomy, Madison, WI.

20. Kohn, L. M. 1979. Delimitation of the economically important plant pathogenic Sclerotinia species. Phytopathology 69:881-886.

21. Little, T. M., and Hills, F. J. 1978. Agricultural Experimentation. John Wiley \& Sons, New York.

22. Liu, L. X., Hsiang, T., Carey, K., and Eggens, J. L. 1995. Microbial populations and suppression of dollar spot disease in creeping bentgrass with inorganic and organic amendments. Plant Dis. 79:144-147.

23. Lo, C.-T., Nelson, E. B., and Harman, G. E. 1996. Biological control of turfgrass diseases with a rhizosphere competent strain of Trichoderma harzianum. Plant Dis. 80:736-741.

24. Lo, C.-T., Nelson, E. B., and Harman, G. E. 1997. Improved biocontrol efficacy of Trichoderma harzianum 1295-22 for foliar phases of turf diseases by use of spray applications. Plant Dis. 81:1132-1138.

25. MacDonald, W. L., and Fulbright, D. W. 1991. Biological control of chestnut blight: Use and limitations of transmissible hypovirulence. Plant Dis. 75:656-661.

26. Melzer, M., and Boland, G. J. 1995. Transmissible hypovirulence in Sclerotinia minor. Can. J. Plant Pathol. 18:19-28.

27. Nelson, E. B., and Craft, C. M. 1991. Introduction and establishment of strains of Enterobacter cloacae in golf course turf for the biological control of dollar spot. Plant Dis. 75:510-514.

28. Nelson, E. B., and Craft, C. M. 1992. Suppression of dollar spot on creeping bentgrass and annual bluegrass turf with compost-amended topdressings. Plant Dis. 76:954-958.

29. Nuss, D. L., and Koltin, Y. 1990. Significance of dsRNA genetic elements in plant pathogenic fungi. Annu. Rev. Phytopathol. 28:37-58.

30. Polashock, J. J., Anagnostakis, S. L., Milgroom, M. G., and Hillman, B. I. 1994. Isolation and characterization of a virus-resistant mutant of Cryphonectria parasitica. Curr. Genet. 26:528-534.

31. Rodriguez, F., and Pfender, W. F. 1997. Antibiosis and antagonism of Sclerotinia homoeocarpa and Drechslera poae by Pseudomonas fluores- 
cens Pf-5 in vitro and in planta. Phytopathology 87:614-621.

32. Smiley, R. W., Dernoeden, P. H., and Clarke, B. B. 1992. Compendium of Turfgrass Diseases. 2nd ed. The American Phytopathological Society, St. Paul, MN.

33. Sneh, B., Ichielevich-Auster, M., and Plaut, Z. 1989. Mechanisms of seedling protection induced by a hypovirulent isolate of Rhizoctonia solani. Can. J. Bot. 67:2135-2141.

34. Sonoda, R. 1989. Vegetative compatibility groups among Sclerotinia homoeocarpa from leaves of Paspalum notatum. Proc. Soil Crop Sci. Soc. Fla. 48:35-36.

35. Van Alfen, N. K. 1982. Biology and potential for disease control of hypovirulence of Endothia parasitica. Annu. Rev. Phytopathol. 20: 349-362.

36. Vargas, J. M. 1981. Management of Turfgrass Diseases. Burgess Publishing, Co., Minneapolis, MN.

37. Zhou, T., and Boland, G. J. 1993. Hypovirulence and double-stranded RNA in Sclerotinia homoeocarpa. (Abstr.) Phytopathology 83:1347.

38. Zhou, T., and Boland, G. J. 1995. Vegetative compatibility and transfer of double stranded RNA in Sclerotinia homoeocarpa. (Abstr.) Can. J. Plant Pathol. 17:365.

39. Zhou, T., and Boland, G. J. 1997. Hypovirulence and double-stranded RNA in Sclerotinia homoeocarpa. Phytopathology 87:147-153. 